\title{
ACESSO DA POPULAÇÃO A MEDICAMENTOS DURANTE A PANDEMIA DO NOVO CORONAVÍRUS
}

Access of the population to medicines during the pandemic of the new coronavirus

Acceso de la población a medicamentos durante la pandemia del nuevo coronavirus

"Artigo premiado no V Congresso Internacional de Atenção Primária à Saúde-V CIAPS, realizado em Teresina-PI, em 2021"

Maria Gabriela da Costa Lacerda • Discente • Universidade Federal do Piauí-UFPI - E-mail: gabilacerda01@hotmail.com

Amália Roberta de Morais Barbosa • Farmacêutica • Universidade Federal do Piauí - E-mail: amaliarobertamb@gmail.com

Carla Solange de Melo Escórcio Dourado • Docente • Universidade Federal do Piauí • E-mail: csmdourado@gmail.com

Autora correspondente:

Maria Gabriela da Costa Lacerda • E-mail: gabilacerda01@hotmail.com 


\section{ciêncíncia
puúl}

\section{RESUMO}

Introdução: Em 2020 a Organização Mundial da Saúde declarou a pandemia do novo coronavírus. Diante desse cenário vários estudos começaram a ser realizados em busca de uma terapia eficaz para o manejo clínico dos pacientes. A Cloroquina e a Hidroxicloroquina foram os primeiros medicamentos testados. A divulgação dos resultados iniciais fez aumentar a procura desses medicamentos em farmácias e drogarias. Objetivo: Avaliar o acesso da população a medicamentos na pandemia e o uso das "promessas terapêuticas": Cloroquina, Hidroxicloroquina e Ivermectina para prevenção e tratamento da COVID-19. Metodologia: Trata-se de um estudo descritivo exploratório de abordagem quantitativa, não probabilístico e por conveniência. A coleta de dados foi realizada online, via Google Formulários. Participaram 1.754 pessoas, resultando em 1.748 questionários válidos. A amostra foi distribuída em 3 grupos, de acordo com a pergunta de $\mathrm{n}^{\circ} 11$ do formulário de pesquisa: "Você já teve COVID-19?". Resultados: Dos 1.748 respondentes, 200 (11,4\%) pertenciam ao grupo que “teve COVID-19", 1.041 (59,6\%) ao grupo que não teve a doença, e 507 (29\%) responderam não saber se foram infectados. No que diz respeito ao acesso a medicamentos na pandemia, 55,2\% do total da amostra relatou não ter sido afetado, e $29 \%$ disseram ter tido o acesso afetado de alguma forma. Em relação ao uso das "promessas terapêuticas", $61 \%$ dos respondentes disse não ter feito uso com finalidade de prevenção, e sim para tratamento, já $52,6 \%$ da população do estudo disse que não fez uso de jeito nenhum, e $46,2 \%$ relatou que fez uso dos medicamentos mencionados para tratar a COVID-19. Conclusões: Constatou-se que a explosão na busca por medicamentos durante a pandemia não afetou o acesso da população. Além disso, a Cloroquina e a Hidroxicloroquina, não foram amplamente utilizadas para prevenção da doença.

Palavras-Chave: Coronavírus; Pandemia; Farmácia; Automedicação

\section{ABSTRACT}

Introduction: In 2020 the World Health Organization declared the new coronavirus pandemic. In view of this scenario, several studies began to be carried out in search of an effective therapy for the clinical management of patients. The release of initial results has increased demand for Chloroquine and Hydroxychloroquine in pharmacies and drugstores. Objective: To assess the population's access to medicines in the pandemic and the use of "therapeutic promises": Chloroquine, Hydroxychloroquine and Ivermectin for the prevention and treatment of COVID-19. Methodology: Exploratory descriptive study with a quantitative approach, nonprobabilistic and for convenience. Data collection was performed online, via Google Forms. 1,754 people participated, resulting in 1,748 valid questionnaires. The sample was divided into 3 groups, according to question \#11 of the survey form: "Have you ver had COVID-19?". Results: Of the 1,748 respondents, $200(11.4 \%)$ belonged to the roup that "had COVID-19", 1,041 (59.6\%) to the group that did not have the disease, $507(29 \%)$ answered not knowing if they were infected. With regard to access to dicines in the pandemic, 55.2\% of the total sample reported not being affected, and said their access was affected in some way. Regarding the use of "therapeutic 


\section{ciênciála
pural}

promises", $61 \%$ of respondents said they did not use it for prevention purposes, but for treatment, while $52.6 \%$ of the study population said they did not use it at all, and $46,2 \%$ used the medications mentioned to treat COVID-19. Conclusions: It was found that the explosion in the search for medicines during the pandemic did not affect the population's access. Furthermore, Chloroquine and Hydroxychloroquine have not been widely used for disease prevention.

Keywords: Coronavirus; Pandemic; Pharmacy; Self-medication

\section{RESUMEN}

Introducción: En 2020, la Organización Mundial de la Salud declaró la nueva pandemia de coronavirus. Ante este escenario, se comenzaron a realizar varios estudios en busca de una terapia eficaz para el manejo clínico de los pacientes. La publicación de los resultados iniciales ha aumentado la demanda de cloroquina e hidroxicloroquina en farmacias y droguerías. Objetivo: Evaluar el acceso de la población a medicamentos en la pandemia y el uso de "promesas terapéuticas": cloroquina, hidroxicloroquina e ivermectina para la prevención y tratamiento de COVID-19. Metodología: Se trata de un estudio descriptivo exploratorio con enfoque cuantitativo, no probabilístico y por conveniencia. La recopilación de datos se realizó en línea, a través de Google Forms. Participaron 1.754 personas, resultando 1.748 cuestionarios válidos. La muestra se dividió en 3 grupos, de acuerdo con la pregunta \# 11 del formulario de la encuesta: “¿Alguna vez ha tenido COVID-19?”. Resultados: De los 1.748 encuestados, 200 (11,4\%) pertenecían al grupo que "tenía COVID-19", $1.041(59,6 \%)$ al grupo que no tenía la enfermedad y $507(29 \%)$ respondieron sin saber si estaban infectados. Con respecto al acceso a medicamentos en la pandemia, el 55,2\% del total de la muestra informó no estar afectado y el $29 \%$ dijo que su acceso se vio afectado de alguna manera. Con respecto al uso de "promesas terapéuticas", el 61\% de los encuestados dijo que no lo usaba con fines de prevención, sino de tratamiento, mientras que el 52,6\% de la población del estudio dijo que no lo usaba en absoluto, y el 46,2\% informó que utilizaron los medicamentos mencionados para tratar COVID19. Conclusiones: Se encontró que la explosión en la búsqueda de medicamentos durante la pandemia no afectó el acceso de la población. Además, la cloroquina y la hidroxicloroquina no se han utilizado ampliamente para la prevención de enfermedades.

Palabras clave: Coronavirus; Pandemia; Farmacia; Automedicación

\section{ntrodução}

Ao final de 2019 a província de Hubei, na China, torna-se epicentro do surto do vo coronavírus, denominado posteriormente pelos cientistas de SARS-CoV-2, sando a doença denominada de COVID-19. Em janeiro do ano seguinte a anização Mundial da Saúde (OMS) confirma a circulação do vírus. Dias após a 
confirmação países já reportavam a importação do vírus, que espalhou-se rapidamente por vários países do mundo, infectando milhares de pessoas¹.

A alta capacidade de transmissão, associado ao grande número de casos graves, e a inexistência de terapêutica comprovadamente eficaz, levou a condução de vários estudos na tentativa de encontrar uma alternativa terapêutica para o manejo clínico dos pacientes ${ }^{2}$. As pesquisas, ainda em fase inicial, demonstraram que medicamentos como os antimaláricos, Cloroquina $(\mathrm{Cl})$ e Hidroxicloroquina (HCQ), além de antihelmínticos, como ivermectina e nitazoxanida, inibiram fortemente a replicação de uma variedade de RNA (incluindo SARS-CoV) e vírus de DNA nos ensaios in-vitro ${ }^{3}$. A partir, da divulgação dos resultados alcançados, os medicamentos citados ganharam status de "promessas terapêuticas", havendo ampla divulgação de matérias relacionadas as formas de tratamento da COVID-194.

No Brasil, estava no centro da discussão o uso desses medicamentos para "tratamento precoce" e sua introdução no chamado "kit-COVID". Em março, foi publicado, pelo Ministério da Saúde, nota informativa justificando o uso da cloroquina no tratamento da COVID-19. Entretanto, ensaios clínicos randomizados, demonstraram que o uso dos medicamentos não era universalmente eficaz e seguro 5 . Mesmo com a divulgação dos novos resultados, HCQ e ivermectina, por exemplo, ganharam contornos de credibilidade tendo seu uso incentivado nas mídias sociais por médicos, demais profissionais da saúde, agentes públicos, como ministros de Estado e até mesmo, o próprio Ministério da Saúdé6.

Todavia, até o presente momento ainda não há qualquer terapêutica farmacológica eficaz para o tratamento da COVID-19, o que tem motivado a condução de diversos estudos clínicos em busca de medicamentos eficazes contra o SARS-CoV27. Nesse sentido, vários países e organizações de saúde do mundo todo têm criado protocolos clínicos e diretrizes terapêuticas para orientação da prática clínica ${ }^{8,9}$.

Um exemplo de iniciativa no Brasil para acelerar o desenvolvimento de armacos e combater o SARS-CoV-2 é o ensaio clínico Solidariedade (Solidarity) um jeto da OMS e coordenado pela Fundação Oswaldo Cruz (Fiocruz). A iniciativa tem o objetivo investigar a eficácia de quatro tratamentos para a COVID-19, será 
implementada em 18 hospitais de 12 estados, com o apoio do Departamento de Ciência e Tecnologia (DECIT) do Ministério da Saúde. O estudo Solidarity é uma conjugação de esforços em todo o mundo para dar uma resposta rápida sobre quais medicamentos são eficazes no tratamento da COVID-19 e quais são ineficazes e não devem ser utilizados. A resposta a essas perguntas exige que milhares de pacientes participem dos testes das drogas 10 .

Vale destacar que segundo estimativas da OMS cerca de metade dos medicamentos são prescritos, dispensados e vendidos incorretamente ${ }^{11}$, sendo o uso incorreto de medicamentos um dos maiores problemas de saúde pública em todo mundo. Além disso, durante a atual pandemia, a falta de medicamentos eficazes, a rápida veiculação de informações sobre possíveis alternativas terapêuticas e a cultura medicalizadora foram importantes agravantes desse panorama,12.

Diante desse contexto, o objetivo desse estudo foi avaliar o acesso da população a medicamentos na pandemia e o uso das "promessas terapêuticas" (Cloroquina, HCQ e Ivermectina) pela população para prevenção e tratamento da COVID-19.

\section{Metodologia}

Trata-se de um estudo descritivo exploratório de abordagem quantitativa, não probabilístico e por conveniência, cuja coleta de dados foi realizada online via plataforma Google Formulários. Participaram 1.754 respondentes, dos quais 1.752 aceitaram participar voluntariamente, após receberem informações pertinentes ao estudo, por meio da assinatura eletrônica do Termo de Consentimento Livre e Esclarecido (TCLE). Desses, 1.748 questionários foram considerados válidos de acordo com os critérios de inclusão (maiores de 18 anos e residentes no Brasil).

O questionário adotado tinha 37 questões e foi do tipo estruturado não disfarçado, ou seja, o respondente foi informado sobre o objetivo antes do início da pesquisa, através do TCLE e do convite que recebeu para participar. O convite para articipação foi enviado por meio dos aplicativos WhatsApp, Facebook e Instagram, e avés do e-mail institucional da pesquisadora. 
Após a finalização da coleta de dados, as respostas dos questionários válidos foram exportadas para uma planilha do software Microsoft Excel versão 2019 MSO para guarda dos dados. Para facilitar a apresentação dos dados a amostra foi distribuída em 3 grupos (sim, não e não sei) de acordo com a pergunta de número 11 do questionário: “Você já teve COVID-19?”. As associações/relações entre as variáveis foram obtidas pelo teste Qui-quadrado. O nível de significância estabelecido foi de $5 \%(p<0.05)$ e o nível de confiança adotado foi de $95 \%$.

Em consonância com a resolução CNS 466/12, item II.2, o presente estudo foi submetido ao Comitê de Ética em Pesquisa da Universidade Federal do Piauí (UFPI), sendo aprovado sob o parecer $n^{\circ} 4.343 .471$.

\section{Resultados}

A amostra desse estudo foi constituída por 1.748 (100\%) respondentes, sendo que 200 (11,4\%) pertenciam ao grupo que “teve COVD-19”, 1.041 (59,6\%) ao grupo que não teve a doença, e 507 (29\%) responderam não saber se foram infectados. Indagados sobre como se deu o acesso e os motivos que afetaram a aquisição dos medicamentos em meio a pandemia, observamos que o acesso não foi totalmente afetado, uma vez que $55,2 \%$ dos participantes disseram não ter tido dificuldade em adquirir seus medicamentos, no entanto, $29 \%$, relatou ter seu acesso afetado. Dos que tiveram o acesso afetado, a maioria mencionou que isso ocorreu devido à dificuldade de encontrar medicamentos que antes eram achados facilmente (Tabela 1).

Tabela 1: Acesso a medicamentos durante a pandemia de COVID-19. Teresina-PI, 2021.

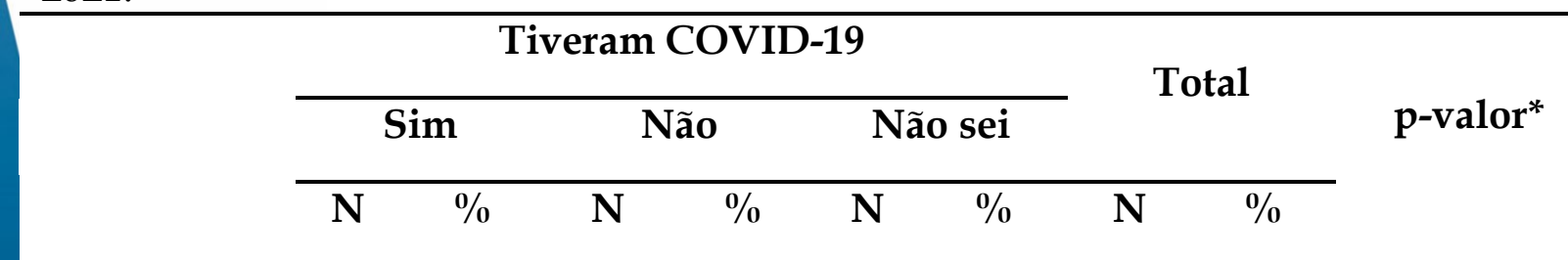

Comprometimento no acesso a

medicamentos

Acesso não foi

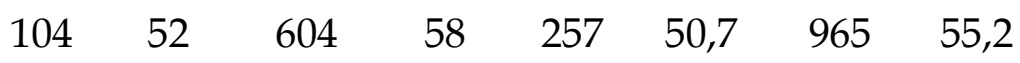


Dificuldade

em encontrar

medicamento

$29 \quad 14,5 \quad 140 \quad 13,4 \quad 82 \quad 16,2 \quad 251 \quad 14,4$

$\mathrm{s}$ achados com

facilidade

Medicamento

encontrado

após muitas

$21 \quad 10,5 \quad 108 \quad 10,4 \quad 46 \quad 9,1 \quad 175 \quad 10$

buscas

Deixou de

tomar por não $11 \quad 5,5 \quad 27 \quad 2,6 \quad 12 \quad 2,4 \quad 50 \quad 2,9$

estar à venda

Deixou de

tomar por não

5

$$
2,5
$$

12

1,2

12

2,4

29

1,7

ter dinheiro

para comprar

Não

souberam

responder

${ }^{*}$ Qui-quadrado Fonte: Autoria Própria

Quanto ao uso dos medicamentos HCQ, Cloroquina, Ivermectina, Azitromicina

e Prednisona para prevenir a infecção pelo vírus, observou-se que 61\% não fez uso desses medicamentos, sendo o grupo que "não teve COVID-19" o que mais concentrou respostas de não uso desses medicamentos (Tabela 2). Dos que responderam terem feito uso ou ter tido algum amigo e/ou familiar, os grupos "não" e "não sei" foram os que concentraram a maioria das repostas para o uso dos medicamentos citados com o objetivo de prevenir a infecção, apesar da divulgação dos resultados dos estudos ientíficos de que esses medicamentos não são seguros eficazes para prevenir a doença abela 2). 
Tabela 2: Uso dos medicamentos Hidroxicloroquina, Cloroquina, Ivermectinina, Azitromicina e Prednisona pelos entrevistados. Teresina-PI, 2021.

Tiveram COVID-19

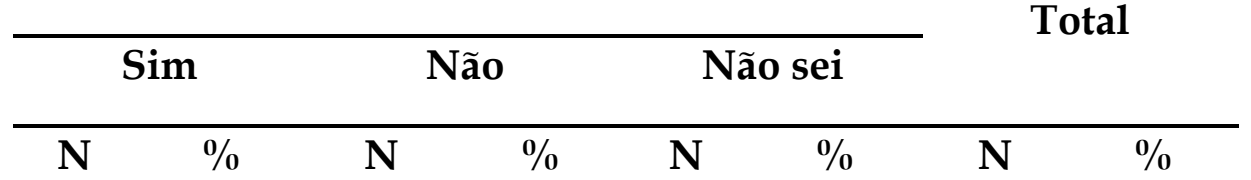

\begin{tabular}{|c|c|c|c|c|c|c|c|c|}
\hline \multicolumn{9}{|l|}{ Prevenção } \\
\hline Fez uso & 103 & 51,5 & 365 & 35,1 & 195 & 38,5 & 663 & 38 \\
\hline Não fez uso & 94 & 47 & 663 & 63,7 & 309 & 60,9 & 1.066 & 61 \\
\hline $\begin{array}{l}\text { Outros } \\
\text { medicamentos }\end{array}$ & 3 & 1,5 & 13 & 1,2 & 3 & 0,6 & 19 & 1 \\
\hline \multicolumn{9}{|l|}{ Tratamento } \\
\hline Fez uso & 168 & 84 & 412 & 39,6 & 228 & 45 & 808 & 46,2 \\
\hline Não fez uso & 30 & 15 & 614 & 59 & 276 & 54,4 & 920 & 52,6 \\
\hline $\begin{array}{l}\text { Outros } \\
\text { medicamentos }\end{array}$ & 2 & 1 & 15 & 0,9 & 3 & 0,2 & 20 & 1,1 \\
\hline
\end{tabular}

Fonte: Autoria Própria

Quando perguntados se fizeram uso de HCQ, Cloroquina, Ivermectinina, Azitromicina e Prednisona para o tratamento, 52,6\% dos respondentes afirmaram que não, sendo que o grupo "não teve" e o grupo "não sabe se teve COVID-19" representaram a maioria das respostas, ainda $46,2 \%$ afirmou ter feito uso ou que amigos e/ ou familiares os utilizaram com esse fim, demonstrando uma diferença bem menor entre o "não uso" e o "uso" (Tabela 2). O grupo que teve COVID-19 em sua grande maioria usou os fármacos mencionados para tratar a doença.

\section{iscussão}

O contexto da pandemia do novo coronavírus fez com que a população uscasse de forma desenfreada e irracional por tratamentos, fazendo com que a venda medicamentos ditos "promissores" aumentasse consideravelmente ${ }^{13}$. Um 
levantamento realizado de janeiro a março de 2020, a pedido dos Conselhos Regionais de Farmácia constatou que os medicamentos associados à prevenção, cura ou alívio dos sintomas da COVID-19, o chamado "kit COVID", tiveram uma explosão de vendas no país, quando comparado com o mesmo período do ano anterior ${ }^{14}$.

Em junho de 2020 a venda de caixas de Ivermectina superou o volume de vendas de todo o ano de 2019, HCQ apresentou um aumento de 67,93\% nas vendas8,9. Foi constatado ainda aumento nas vendas de vitamina C e D, de $180 \%$ e 35,56\%, respectivamente, e ainda aumento nas vendas de paracetamol e dipirona que aumentaram $77,35 \%$ e $54,56 \%$, respectivamente ${ }^{15}$.

Apesar do aumento na procura por medicamentos, medidas foram imediatamente tomadas para evitar o desabastecimento. Os Serviços Farmacêuticos (SF) diante da declaração de emergência de saúde pública declarada pela OMS reforçaram o estoque de medicamentos de antipiréticos, antivirais, antibióticos, dentre outros, já prevendo um aumento na demanda e consumo ${ }^{16}$. Outra medida tomada frente ao aumento da demanda, foi a inclusão de medicamentos como $\mathrm{HCQ}, \mathrm{Cl}$ e Ivermectina na lista de substâncias sujeitas à Receita de Controle Especial17.

Essas atitudes contribuíram para que a população fosse minimamente afetada frente ao aumento do consumo de fármacos na pandemia. Fato que foi observado nesse estudo, uma vez que, mais da metade da amostra relatou não ter encontrado dificuldades no acesso a medicamentos e dos que relataram ter problemas com o acesso esse se deu pela dificuldade em encontrar medicamentos que eram comprados com facilidade (Tabela 1).

Outro fenômeno associado ao aumento da procura e venda está o uso de alguns medicamentos supracitados no texto para prevenção e tratamento da infecção pelo SARS-CoV-2, seja na forma da automedicação ou através da prescrição médica, mesmo sem que estudos robustos comprovassem a eficácia e a efetividade clínica deles ${ }^{15}$. Jossos resultados apontaram que boa parte dos participantes da pesquisa não fizeram so das "promessas terapêuticas" para fins de prevenção ou tratamento (Tabela 2).

De acordo com a OMS, entende-se que há Uso Racional de Medicamento (URM) ando pacientes recebem medicamentos para suas condições clínicas em doses 
adequadas às suas necessidades individuais, por um período adequado e ao menor custo para si e para a comunidade ${ }^{11}$. No entanto, ao observarmos os achados do nosso estudo, especificamente em relação ao tratamento para a COVID-19, percebe-se que a diferença entre o percentual de pessoas que fizeram uso daquelas que não o fizeram é pequena $(6,4 \%)$ (Tabela 2), revelando que os medicamentos foram amplamente utilizados, quer seja por automedicação ou por prescrição médica. Tal situação é razoável para o começo da pandemia, onde estudos preliminares estavam sendo feitos e haviam muitas incertezas no manejo clínico adequado dos pacientes e pânico por parte da população.

No que tange ao URM, o uso de medicamentos para tratar outras doenças, utilizados de forma inequívoca por pacientes que apresentam sintomas do Sars-CoV2, poderá trazer altos riscos à saúde da população. Além de fazer com que aqueles medicamentos possam faltar para os pacientes que realmente necessitam para tratar uma doença crônica específica, baseado em protocolos clínicos e diretrizes terapêuticas ${ }^{18}$.

Outrossim a prescrição off-label, uso de medicamentos não regulamentados para o tratamento da condição para a qual foi prescrito; e o uso compassivo, ou seja, a prescrição quando o medicamento ainda está em fase de teste, indisponível para comercialização, foram duas práticas clínicas comuns durante a pandemia ${ }^{19}$. Vale ressaltar que o aumento das prescrições off-label de Ivermectina foi fortemente influenciado devido aos resultados favoráveis de um estudo in vitro, o demonstrou o impedimento da replicação do SARS-CoV-2, todavia, a dose utilizada no estudo foi dez vezes a aprovada pelas agências reguladoras como ANVISA e FDA, e vem sendo apontado na literatura o alto risco de viés do estudo ${ }^{20,21}$.

Nesse contexto, diversos estudos têm sido conduzidos para avaliação do "KIT COVID", porém, até o momento não há evidências de benefícios clínicos que validem prescrições racionais de Ivermectina fora dos ensaios clínicos randomizados, tendo seu off-label fortemente contraindicado pela OPAS/OMS22. Quanto à droxicloroquina e cloroquina, por exemplo, podem causar inúmeros efeitos laterais de moderados a graves, dentre eles hipoglicemia, retinopatia, arritmia e 
infarto $^{23}$, o que reforça a necessidade de URM e acompanhamento médico. Além disso, não são recomendadas associações como lopinavir/ritonavir e hidroxicloroquina/azitromicina fora de ensaios clínicos randomizados, especialmente, devido ao alto risco de toxicidade e potenciais interações medicamentosas 23 .

Todavia, a manutenção da conduta de usar os fármacos apontados, meses após o início da pandemia no país, uma vez que a pesquisa foi realizada em outubro de 2020, e com evidências científicas apontando para a falta de benefício clínico destes e para os riscos de reações adversas, inclusive perigosas, é intrigante e questionável24. Fatores que podem explicar esse comportamento são, o movimento de infodemia, levando ao surgimento de rumores e desinformação, com manipulação das informações de forma duvidosa; a negação da ciência e a insistência do governo brasileiro em incentivar a sua utilização ${ }^{25}$.

\section{Conclusões}

Constatou-se que diante do cenário de muitas incertezas quanto a tratamentos e métodos eficazes contra o SARS-CoV-2, as "promessas terapêuticas" foram utilizadas como prevenção e amplamente prescritas para o tratamento da doença, evidenciando ser necessário mais investimentos em pesquisas científicas para que se possa o quanto antes chegar ao desenvolvimento de fármacos seguros, eficazes e de qualidade comprovada para a prevenção e o tratamento contra a COVID-19. No entanto, percebeu-se também que apesar de tudo, a sociedade brasileira não sofreu grandes impactos no acesso a medicamentos durante a pandemia, pois as atitudes tomadas para combater o desabastecimento foram eficazes.

\section{Referências}

1. Lana RM, Coelho FC, da Costa Gomes MF, Cruz OG, Bastos LS, Villela DAM, codeço CT. Emergência do novo coronavírus (SARS-CoV-2) e o papel de uma igilância nacional em saúde oportuna e efetiva. Cad. Saúde Pública. 2020; 36(3).

Paiva AM, Pinto AWS, Cançado BL, Chequer FMD, Pereira ML, Baldoni AO. Efeito "promessas terapêuticas" sobre os preços de medicamentos em tempos de demia. J. Health Biol Sci. 2020; 8(1): 1-5. 
3. Paumgartten FJR, Oliveira ACAX de. Off label, compassionate and irrational use of medicines in COVID-19 pandemic, health consequences and ethical issues. Ciênc. saúde coletiva, 2020; 25(9) : 3413-3419.

4. Menezes CR, Sanches C, Chequer FMD. Efetividade e toxicidade da cloroquina e da hidroxicloroquina associada (ou não) à azitromicina para tratamento da COVID-19. O que sabemos até o momento?. J. Health Biol Sci. 2020; 8(1) : 1-9.

5. Lotfi M, Hamblin MR, Rezaei, N. COVID-19: Transmission, prevention, and potential therapeutic opportu-nities. Clinic. Chim. Act., 2020; 508 : 254-266.

6. Melo JRR, Duarte EC, de Moraes MV, Fleck K, Arrais PSD. Automedicação e uso indiscriminado de medicamentos durante a pandemia da COVID-19. Cad. Saúde Pública. 2021; 37(4).

7. Clinical Trials [Internet]. Studies found for: COVID-19, search results: 1324; [citado 29 de setembro de 2021]. Disponível em: https://clinicaltrials.gov/ct2/results?cond=COVID-19

8. Brasil [Internet]. Protocolo de Manejo Clínico para o coronavírus (2019-nCoV) Versão 9. Brasília: Ministério da Saúde, Secretaria de Atenção à Saúde, SAPS [2020 maio; citado 29 de setembro de 2021]. Disponível em: http:// portalarquivos2.saude.gov.br/images/pdf/2020/fevereiro/11/ protocolomanejo-coronavirus.pdf

9. National Institutes of Health [Internet]. COVID-19 Treatment Guidelines Panel. Coronavirus Diseases 2019 (COVID-19) Treatment Guidelines [citado 29 de setembro de 2021]. Disponível em: https://www.covid19treatmentguidelines.nih.gov/introduction/

10. Fiocruz - Fundação Oswaldo Cruz [Internet] [citado 26 de setembro de 2021]. Disponível em: https:/ /agencia.fiocruz.br/ node/12299.

11. WHO. Promoting rational use of medicines: core components. Promoción del uso racional de medicamentos: componentes centrale; 2002 [citado 26 de setembro de 2021]. Disponível em: https://apps.who.int/iris/handle/10665/67438

12. Camargo Jr KRD. Medicalização, farmacologização e imperialismo sanitário. Cad Saúde Pública. 2013; 29:844-6.

13. Rome BN, Avorn J. Drug Evaluation during the Covid-19 Pandemic. N Engl J Med. 2020 Abr; 382(24): 2282-84. doi: 10.1056/NEJMp2009457.

Lima WG, Cardoso BG, Simião DC, Amorim JM, Silva CA, Brito JCM. Uso acional de medicamentos e plantas medicinais contra a COVID-19 (SARS-CoVUm problema emergente. Braz. J. H. Pharm. 2020; 2(3). 
15. Conselho Federal de Farmácia (CFF) [Internet]. Levantamento mostra como o medo da Covid-19 impactou venda de medicamentos. [citado 15 de junho de 2021] Disponível em: http://covid19.cff.org.br/levantamento-mostra-como-o-medo-dacovid-19-impactou-venda-de-medicamentos/

16. Pereira MP, Oliveira R, Batista M, Cardoso J, Vilaverde R, Cabeças T, et al. Os Serviços Farmacêuticos CUF Infante Santo no Combate à Pandemia COVID-19: Dias Difíceis, Dedicação Incondicional. Gazeta Médica. 2020; 2(7).

17. Brasil [Internet]. Ministério da Saúde. Anvisa. Resolução de Diretoria Colegiada RDC $N^{\circ}$ 405, de 22 de julho de 2020. [citado 16 de junho de 2021] Disponível em: https://www.in.gov.br/en/web/dou/-/resolucao-de-diretoria-colegiada-rdc-n-405de-22-de-julho-de-2020-268192342.

18. Brasil [Internet]. Ministério da Saúde. Uso racional de medicamentos; 2007 [citado 25 de setembro de 2021]. Disponível em: https:/ / bit.ly/2K8rZy7

19. American Cancer Society [Internet]. Compassionate Drug Use; 2018 [citado 29 de setembro de 2021]. Disponível em: https://www.cancer.org/treatment/treatmentsand-sideeffects/clinical-trials/compassionate-drug-use.html

20. Caly L, Druce JD, Catton MG, Jans DA, Wagstaff KM. The FDA-approved drug ivermectin inhibits the replication of SARS-CoV-2 in vitro. Antiviral Res. 2020 178:104787.

21. Schmith VD, Zhou J (Jessie), Lohmer LRL. The Approved Dose of Ivermectin Alone is not the Ideal Dose for the Treatment of COVID-19. Clin Pharmacol Ther. 2020.

22. OPAS [Internet]. Recomendação sobre o uso de ivermectina no tratamento de COVID-19. OPAS; 2020 [citado 29 de setembro de 2021]. Disponível em: https:/ /iris.paho.org/ bitstream/handle/10665.2/52462/OPASIMSCDECOVID19200033_por.pdf? sequence $=1 \&$ isAllowed $=y$

23. Sahraei Z, Shabani M, Shokouhi S, Saffaei A. Aminoquinolines against coronavirus disease 2019 (COVID-19): chloroquine or hydroxychloroquine. Int J Antimicrob Agents. 2020.

24. Gérad A, Romani S, Fresse A, Viard D, de Nadège G, Granvuillemin A, et al. "Offlabel" use of hydroxychloroquine, azithromycin, lopinavir-ritonavir and chloroquine in COVID-19: A survey of cardiac adverse drug reactions by the French Network of Pharmacovigilance Centers. Therapie. 2020 jul - agosto; 75(4):371-379.

Santos-Pinto CDuB, Miranda ES, Osorio-de-Castro CGS. O "kit-covid" e o grama Farmácia Popular do Brasil. Cad. Saúde Pública. 2021; 37(2). 